\title{
Observational Study Sampling Method
}

National Cancer Institute

\section{Source}

National Cancer Institute. Observational Study Sampling Method. NCI Thesaurus. Code C126067.

The process by which subjects are selected to participate in observational studies. 